\title{
Sparking consciousness
}

By fostering functional neural activation, deep brain stimulation (DBS) led a man to regain communication after spending six years in a minimally conscious state, according to a recent case report in Nature $(448,600-603)$.

After a severe traumatic injury, neural circuits can be disrupted. Widespread tissue damage disconnects the brain from responding to the environment, and the patient remains permanently unconscious. If the damage is less severe, however, some individuals occasionally have voluntary movements and wakefulness, experiencing what is called a 'minimally conscious state'. These activities indicate that in these individuals, there is some preservatioin of neural circuitry.

DBS, in which electrodes are implanted into the brain to directly stimulate neuron activity, has been used successfully to improve the pacemaking of neurons and to reinforce weakened neuronal circuits. In their case report, Nicholas Schiff et al. proposed that stimulation of the thalamus, which connects the brainstem and the cortex, would strengthen communication between the arousal and cognitive networks, and so consistently conscious behaviour and speech could be recovered.

Schiff et al. tested their hypothesis in a 38-year-old man who had been in a minimally conscious state for 6 years. Although his spontaneous episodes of wakefulness and communication were unpredictable, neurological tests revealed some cerebral activity that might be tapped if better neural functional connectivity were established. The investigators implanted electrodes into the man's thalamus and began to search for stimulation parameters that would increase the frequency of the man's conscious behavior.

Schiff et al. assessed his awareness by examining his ability to respond to commands and to perform basic tasks, such as naming objects and feeding himself. They observed dramatic improvement in every test at different times across the study and these recovered behaviors correlated with the periods of stimulation.

This case report suggests that deep brain stimulation could be used to strengthen neural networks that are inconsistently active owing to injury, but a word of caution is necessary. In addition to being a single case, this particular man had significant preservation of brain structure, including the language centers. Many patients with more extensive lesions may not respond as well. Nonetheless, the lessons learned from this study may help to identify individuals who could best benefit from DBS, and may offer a therapy that could allow these patients to reconnect with the world.

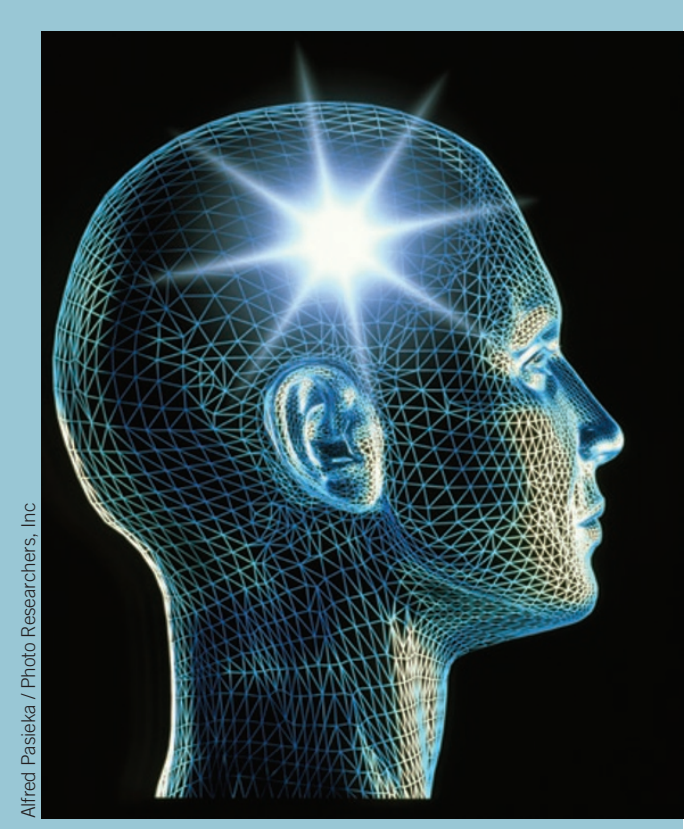
-Katherine Stevens 